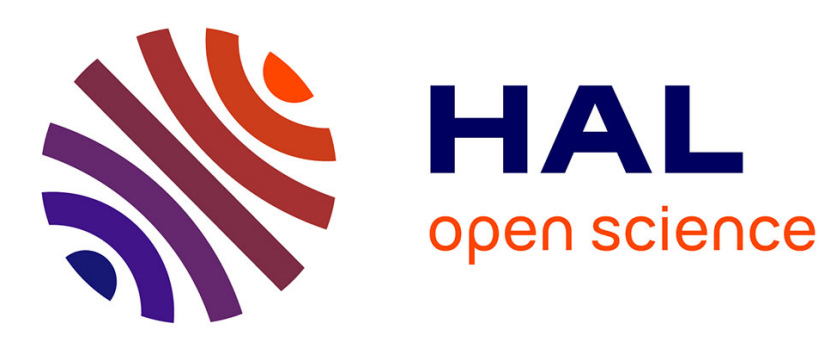

\title{
A Three-Step Scenario Involved in Particle Capture on a Pore Edge
}

\author{
Paul Duru, Yannick Hallez
}

\section{To cite this version:}

Paul Duru, Yannick Hallez. A Three-Step Scenario Involved in Particle Capture on a Pore Edge. Langmuir, 2015, vol. 31 ( $\mathrm{n}^{\circ}$ 30), pp. 8310-8317. 10.1021/acs.langmuir.5b01298 . hal-01223261

\section{HAL Id: hal-01223261 \\ https://hal.science/hal-01223261}

Submitted on 2 Nov 2015

HAL is a multi-disciplinary open access archive for the deposit and dissemination of scientific research documents, whether they are published or not. The documents may come from teaching and research institutions in France or abroad, or from public or private research centers.
L'archive ouverte pluridisciplinaire HAL, est destinée au dépôt et à la diffusion de documents scientifiques de niveau recherche, publiés ou non, émanant des établissements d'enseignement et de recherche français ou étrangers, des laboratoires publics ou privés. 


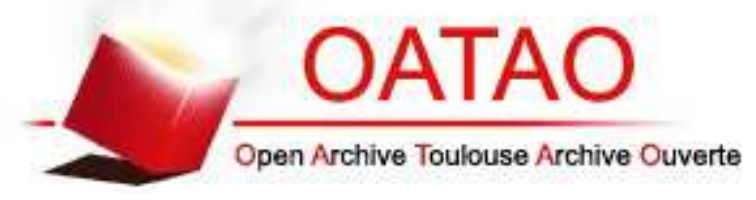

\section{Open Archive TOULOUSE Archive Ouverte (OATAO)}

OATAO is an open access repository that collects the work of Toulouse researchers and makes it freely available over the web where possible.

This is an author-deposited version published in : http://oatao.univ-toulouse.fr/ Eprints ID : 14331

To link to this article : DOI:10.1021/acs.langmuir.5b01298 URL : http://pubs.acs.org/doi/abs/10.1021/acs.langmuir.5b01298

To cite this version : Duru, Paul and Hallez, Yannick A Three-Step Scenario Involved in Particle Capture on a Pore Edge. (2015) Langmuir, vol. 31 ( $\left.{ }^{\circ} 30\right)$. pp. 8310-8317. ISSN 0743-7463

Any correspondance concerning this service should be sent to the repository administrator: staff-oatao@listes-diff.inp-toulouse.fr 


\title{
A Three-Step Scenario Involved in Particle Capture on a Pore Edge
}

\author{
Paul Duru* ${ }^{* \dagger, \ddagger}$ and Yannick Hallez ${ }^{\text {II, }}$ \\ †Université de Toulouse, INPT, UPS, IMFT (Institut de Mécanique des Fluides de Toulouse), Allée Camille Soula, F-31400 \\ Toulouse, France \\ ${ }^{\ddagger}$ CNRS, IMFT, F-31400 Toulouse, France \\ "IIniversité de Toulouse, INPT, UPS, LGC (Laboratoire de Génie Chimique), 118 route de Narbonne, F-31062 Toulouse, France. \\ ${ }^{\S}$ CNRS, LGC, F-31030 Toulouse, France
}

ABSTRACT: A scenario is proposed to describe the capture of a spherical particle around a cylindrical pore. This geometry, "ideal" as far as the problem of particle capture on a filtration membrane is concerned, is clearly relevant in view of the porescale geometry of nucleopore or microsieve filtration membranes, and also of some microfluidic systems used to perform fluid-particle separation. The present scenario consists of three successive steps: particle deposition on the membrane away from the pore, subsequent reentrainment of some of the deposited particles by rolling on the membrane surface, and final arrest by a stabilizing van der Waals torque

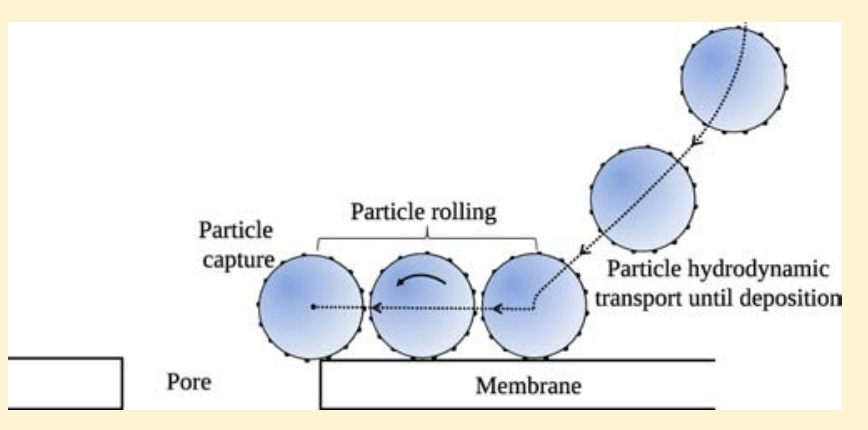
when the particle rolls over the pore edge. A modeling of these three steps requires the hydrodynamic and physicochemical particle-membrane interactions to be detailed close to the singular pore edge region and raises questions concerning the role of particle surface roughness. The relevance and robustness of such of a scenario for rough micrometer-sized latex particles is emphasized and comparisons are made with existing experimental data.

\section{INTRODUCTION}

Particle capture by a collecting surface is a ubiquitous phenomenon, with applications covering an extremely broad spectrum, from environmental flow problems (e.g., collection of aerosol particles by rain droplets ${ }^{1}$ or plankton capture by subaquatic tentacular feeders ${ }^{2}$ ) to industrial applications (e.g., filtration as a fluid cleaning process ${ }^{3}$ ). In the context of filtration, particle capture on porous filtration membranes is known to occur even when the radius of the particle, $a$, is smaller than the pore radius, $r_{\mathrm{p}}$. Various structures can then be observed on the surface of filtration devices, from dendrites to arches "bridging" the pore openings, depending on the physicochemical and hydrodynamic conditions of the filtration process. ${ }^{4-6}$ Several scenarios have been proposed to describe pore bridging, which can ultimately lead to complete pore clogging. Some authors invoke the instantaneous creation of a bridge due to the converging flow (hydrodynamic bridging) 7,8 while others describe its progressive build up by successive particle depositions. ${ }^{4,9,10}$ The deposition of the very first particle close to the pore is an essential step in the latter scenario of pore clogging, and will impact the subsequent particle plug structure. For this reason, the specific case of the capture of a single spherical particle close to a single cylindrical opening in a flat surface, in a situation where there is no length scale separation between the particle and opening sizes, is relevant for filtration applications. Surprisingly, studies of this situation are rare, certainly because this apparently simple geometry brings its share of problems as far as the description of the particle-membrane hydrodynamic and physicochemical interactions are concerned, particularly in the singular pore edge region. ${ }^{10}$ As a matter of fact, the spherical particle/ cylindrical pore geometry also closely matches that encountered in microsieve membranes, that is, microfabricated membranes consisting of a thin $(O(\mu \mathrm{m}))$ silicon sheet patterned by regular arrays of cylindrical holes, the radius of which is typically in the range $O(0.1-10 \mu \mathrm{m})$. To a lesser extent, this geometry is also similar to configurations found in some microfluidic devices where particles moving in a "large" microchannel are flow-driven through a set of smaller channels and possibly captured at their entrance. ${ }^{4,9,11,12}$

The problem of predicting particle capture location on a microsieve has been addressed recently by Lin et al. ${ }^{10}$ Their experimental results highlighted the existence of two wellseparated, preferential locations for particle capture around a cylindrical pore: an "outer" capture position shifted from the pore edge by a fraction of the particle radius and an "inner" capture position with the contact point on the pore edge and the particle center of mass well above the pore opening. These authors attempted to explain these two distinct capture positions with a purely hydrodynamic approach based on an 
accurate description of the trajectory of a particle in the flow and an effective distance for capture criterion intended to model the effects of surface forces: particles passing at a distance smaller than an arbitrary value $\epsilon$ from the membrane were instantaneously and definitively captured at the position where the criterion was satisfied. They observed that unrealistically large values of $\epsilon$ (compared to the range of attractive surface forces) had to be imposed to obtain two distinct capture positions. This conclusion highlighted the need for more elaborate modeling of the particle capture mechanism.

The aim of the present Article is to provide such a modeling strategy by introducing explicit calculations of the van der Waals (vdW) forces near the microsieve, and, in particular, near the pore edge, while retaining Lin et al.'s good description of hydrodynamic interactions. It turns out that explicitely accounting for vdW forces alone in the dynamics of suspended particles is not sufficient for two preferential positions for particle capture to be obtained and that particle rolling on the membrane surface has to be taken into account. These ingredients lead to the simplest three-step scenario giving satisfactory predictions. During the first step, suspended particles are transported toward the microsieve by the flow, with significant influence of both hydrodynamic and vdW particle-membrane interactions. If a particle follows a trajectory leading to contact with the membrane, then, once deposited, it will experience both a hydrodynamic torque, $\Gamma_{\mathrm{h}}$, tending to sweep it toward the pore and a resistive torque, $\Gamma_{a}$ due to adhesion forces. If $\Gamma_{\mathrm{h}}$ overcomes $\Gamma_{\mathrm{a}}$ the particle starts a rolling phase. This is the second step of the scenario. During the third and last step, every rolling particle passes over the pore edge and experiences a strong resistive vdW torque, $\Gamma_{e}$, able to arrest the particle with its center of mass above the pore opening. This torque is generated by the asymmetry of the particle-membrane vdW interaction when the particle is above the pore edge.

The modeling techniques and assumptions associated with each of the three steps are presented in section 2 . The results obtained with the proposed scenario are presented and discussed in section 3. Although the article is mainly based on the hydrodynamic and surface interaction parameters of Lin et al.'s study, some elements are also provided to show that this three-step scenario is quite generic and relevant for a broader range of parameters.

\section{MODEL}

Computing the trajectory of a particle to its final capture location involves both hydrodynamic and surface interactions. Before going into the details of their modeling, the relevant particle and membrane properties are recalled briefly.

2.1. System. In this paper, we will consider the parameters of the experiments by Lin et al., ${ }^{10}$ where latex particles suspended in water (dynamic viscosity $\mu_{\mathrm{f}}=10^{-3} \mathrm{~kg} \mathrm{~m}^{-1} \mathrm{~s}^{-1}$ and mass density $\rho_{\mathrm{f}}=1 \mathrm{~g}$ $\mathrm{cm}^{-3}$ ) were filtered through a silicon-nitride coated microsieve with pores of radius $r_{\mathrm{p}}=5 \mu \mathrm{m}$.

The latex particles have a radius $a \simeq 2.5 \mu \mathrm{m}$ and a mass density $\rho_{\mathrm{p}}=$ $1.05 \mathrm{~g} \mathrm{~cm}^{-3}$. They are known to be covered with a layer of polymer chains which is typically a few nanometer thick, giving the particles a rough character. ${ }^{13,14}$ Scanning electron microscopy (SEM) and atomic force microscopy (AFM) measurements have shown that they are also covered with asperities of much larger sizes. Considine et al. ${ }^{15}$ reported asperities with sizes $a^{\prime}$ in the range $8-20 \mathrm{~nm}$ for $6 \mu$ m diameter surfactant-free sulfate latex particles similar to the ones considered here. Assuming the asperities to be homogeneously distributed over the particle surface, the typical distance, $h$, between two asperities scales as $a^{\prime} / S^{1 / 2}$, where the fractional surface coverage by asperities $S$ has been estimated to be around a few percent. ${ }^{16}$ In the following, we will therefore consider $h \simeq 10 a^{\prime}$. The asperities will be modeled as hemispherical bumps of height $a^{\prime}$ described by a normal distribution with mean value $\mu=15 \mathrm{~nm}$ and standard deviation $\sigma=2 \mathrm{~nm}$, evenly distributed with a spacing $h=150 \mathrm{~nm}$, consistent with measurements of Considine et al. ${ }^{15}$ and Suresh and Walz. ${ }^{16}$

2.2. van der Waals Interactions. In the experimental study by Lin et al., weak particle surface charge and insensitivity of the results to $\mathrm{pH}$ and salinity of the suspending fluid were reported, which suggests that electrostatic effects were negligible. The attractive vdW forces are then the key to describing particle capture in the present system.

The Hamaker additivity hypothesis is adopted throughout this work since the geometry would render other approaches intractable. A Hamaker constant $H=3.7 \times 10^{-20} \mathrm{~J}$ for the polystyrene-water-silicon nitride system is computed with the approximate relation (11.13) of Israelachvili, ${ }^{17}$ from the values for the optical indexes and relative permittivities of each of these three materials.

2.2.1. VdW Force Exerted on a Particle Suspended in the Flow. The vdW interaction between a rough particle and the smooth membrane surface (the subnanometric roughness of the silicon surface will be neglected) can be written as the sum of two terms: the contribution of the smooth spherical core of the particle of radius $a$ and the contribution from all the hemispherical bumps. As will be proved a posteriori, the contribution of the asperities can safely be neglected during the period of time before deposition of the particle. Particles will therefore be modeled as smooth spheres of radius a during step 1 . The problem is, however, not trivial for the singular pore edge/spherical particle geometry of the present study, so the vdW interaction has been computed numerically for a detailed set of locations above the membrane and pore, and on the pore edge.

Precise numerical evaluation of the vdW force including retardation effects would require daunting numerical efforts even for simple geometries, ${ }^{18}$ and is not applicable to the present one. If Hamaker's additivity hypothesis is invoked, the problem is greatly simplified. In this framework, the pairwise interaction force exerted between every pair of atoms of both solids is summed, neglecting any many-body interactions. This approximation usually gives results within 10-20\% of the exact value. The pairwise interaction energy between two atoms is $E=-\beta / l^{6}$, where $\beta$ is the Lifschitz-van der Waals energy constant and $l$ is the distance between the atoms. The pairwise force exerted on an atom at position $A$ by an atom located at position $B$ is $f_{B \rightarrow A}=-\nabla E$ $=6 \beta l^{-8} 1$ where $\mathbf{l}=\mathrm{AB}$. The force density exerted at some point $\mathbf{x}$ in the particle by the entire membrane is

$$
\mathbf{f}(\mathbf{x})=\frac{6 H}{\pi^{2}} \int_{V_{2}} \frac{1}{l^{8}} \mathbf{l} d V_{2}
$$

where $H=\beta Q_{1} Q_{2} \pi^{2}$ is the effective Hamaker constant, $Q_{i}$ is the number of atoms per unit volume in the solid $i$, the index 1 (respectively 2) refers to the particle (respectively the membrane), $\mathbf{l}=$ $\mathbf{x}_{2}-\mathbf{x}$, and $\mathbf{x}_{2}$ is the point scanning the volume of the membrane $V_{2}$. The total force exerted by the membrane on the particle is

$$
\mathbf{F}_{\mathrm{a}}=\int_{V_{1}} \mathbf{f}\left(\mathbf{x}_{1}\right) \mathrm{d} V_{1}
$$

The computation of the vdW force with eq 2 requires the evaluation of a six-dimensional integral of a very steep function. This is a difficult problem both analytically and numerically. Some authors have reduced the number of integration dimensions by converting the volume integrals into surface integrals. This is done, for example, in the surface formulation method ${ }^{19,20}$ and in the surface element integration method. ${ }^{21,22}$

To the best of the authors' knowledge, the interaction force between a sphere and a membrane perforated by a cylindrical pore has never been computed accurately when the sphere is outside the pore, although it has been evaluated approximately for a sphere near a rounded pore in Bowen et al. ${ }^{23}$ and has been evaluated accurately with the definition 2 for a sphere inside a cylindrical pore. ${ }^{24}$ In the present work, the three integrals on the volume of the sphere are performed analytically and only the three integrals on the microsieve volume 
remain. The latter integrals are evaluated with a standard second order quadrature on an adaptive octree mesh. The microsieve volume is iteratively split into small cubes only in regions where the integrand is the largest, to increase the numerical accuracy without increasing the CPU load too much. The force computation is performed in several passes, with the mesh being refined automatically between two successive passes. The process is stopped when the value of the force does not vary by more than $2-3 \%$ between two passes. More details can be found in Hallez. ${ }^{25}$

A continuous model of the vdW force components $F_{a, R}$ and $F_{a, Z}$ for any location along the particle trajectory was obtained by using semianalytical expressions fitting the discrete numerical values obtained from eq 2. This model is an ad hoc modification of the exact expressions for $F_{\mathrm{a}, R}$ and $F_{\mathrm{a}, Z}$ in the limit $a / r_{\mathrm{p}} \rightarrow 0$. The details are given in the Supporting Information.

2.2.2. VdW Torque Exerted on a Particle Deposited Far from the Pore Edge. During the rolling and final arrest steps, the details of the particle surface play a major role by providing the only means to counterbalance the hydrodynamic torque $\Gamma_{\mathrm{h}}$, which tends to sweep the particles from the surface. To compute the adhesion torque, $\Gamma_{a}$ far from the pore edge, the membrane surface is considered to be smooth and flat. The spherical particle located at a radial position $R$ is assumed to lie on two asperities located at positions $R \pm h / 2$ and at a contact distance $d_{c}$ to the membrane surface (for smooth surfaces in contact, Born repulsion is the reason why the distance of closest approach $d_{c}$ is nonzero and in this case $d_{c}$ is typically considered to be $\approx 0.4 \mathrm{~nm}$ ). The norm of the adhesion torque around the contact point of the downstream asperity is then:

$$
\Gamma_{\mathrm{a}}=\frac{h}{2} \frac{H a}{6\left[a^{\prime}-a+\sqrt{a^{2}-h^{2} / 4}+d_{c}\right]^{2}}+h \frac{H a^{\prime}}{6 d_{c}{ }^{2}}
$$

where the first term is the torque due to the adhesion force exerted on the spherical particle core shifted from the surface by the asperities and the second term is the torque due to the upstream asperity in contact with the membrane, both terms being computed with the Derjaguin approximation (contributions of additional asperities located at $R \pm 3$ $h / 2$ do not significantly modify the values obtained from eq 3 ). Such a description is quite generic and is the building block of several adhesion models (e.g., Rumpf ${ }^{26}$ and Rabinovitch et al. ${ }^{27}$ ). The roughness distribution and the model of hemispherical asperities used here can be criticized but it has the merit of involving only three physical parameters: $h, a^{\prime}$, and $d_{c}$, with experimental observations giving reasonable ranges of values for two of them $\left(h\right.$ and $\left.a^{\prime}\right)$. More sophisticated models exist, with non-Gaussian distributions, several length scales, flattened asperities, or including several asperities in contact simultaneously, ${ }^{28-30}$ but they all involve additional parameters which are unknown and would have to be fitted somehow. Therefore, no insight would be gained by using these models here, and we restrict ourselves to the simple model eq 3 , showing it to be sufficient to reproduce the experimental observations.

2.2.3. VdW Torque Exerted on a Particle Deposited on the Pore Edge. A particle will be located at least temporarily on the pore edge either if it has rolled on the membrane toward the pore or if it first touched the membrane directly on the pore edge. In both cases, it experiences a vdW torque opposing the hydrodynamic torque $\Gamma_{\mathrm{h}}$. In fact, the part of the particle remaining above the membrane is still attracted by the membrane whereas the part of the particle hanging above the pore lacks this attraction. This imbalance creates a localized, negative stabilizing adhesion torque of norm $\Gamma_{\mathrm{e}}$. To our knowledge, such an important effect that is expected to appear close to any geometrical singularity has never been highlighted before. Similarly to the adhesion torque $\Gamma_{a}, \Gamma_{\mathrm{e}}$ is expected to depend critically on both the particle and membrane surface properties (physicochemical effects in play, roughness of surfaces, tiny details of the pore edge geometry, etc.). The contribution of the spherical particle core to the torque around the contact point between the sphere and the pore edge $E$, $\Gamma_{\mathrm{e}, \text { core }}$ can be readily obtained (once the vdW interaction has been computed numerically as mentioned before) using the simple relation

$$
\Gamma_{\mathrm{e}, \text { core }}=\mathbf{E G} \times \mathbf{F}_{\mathrm{a}}
$$

as shown in the Supporting Information and where the point $G$ locates the particle center of mass (see Figure 1). The effect of an asperity

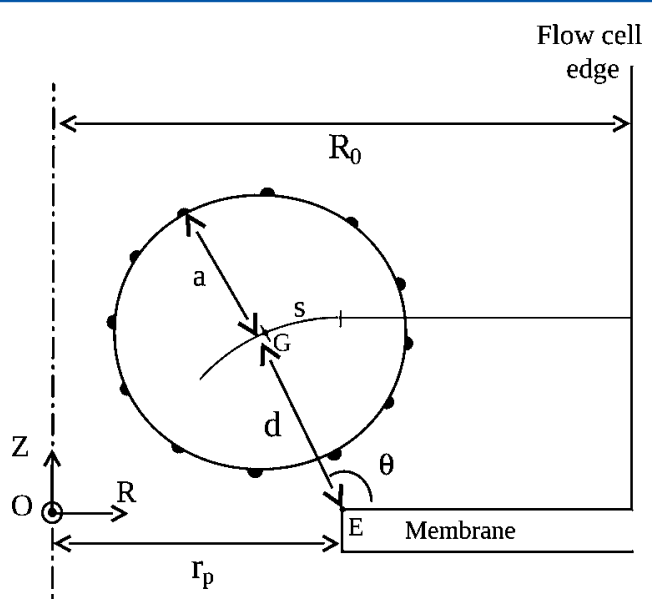

Figure 1. Particle approaching a cylindrical orifice located at the bottom of an axisymmetrical flow cell of radius $R_{0}$. The orthogonal coordinate system $\left(\mathbf{e}_{R}, \mathbf{e}_{\mathrm{Z}}, \mathbf{e}_{R} \wedge \mathbf{e}_{\mathrm{Z}}\right)$ is centered on point $O$. The coordinate $d$ is the distance between the particle center of mass and the membrane. The coordinate $s$ is defined along the iso- $d$ contour shown as a solid line by $s=-d(\theta-\pi / 2)$ for $R<r_{\mathrm{p}}$, and $s=R-r_{\mathrm{p}}$ for $R \geq r_{\mathrm{p}}$. Points $\mathrm{E}$ and $\mathrm{G}$ locate the pore upper edge $\left(R=r_{\mathrm{p}}, Z=0\right)$ and the particle center of mass, respectively.

touching the membrane upstream at a distance $h^{\prime}$ while the particle is on the pore edge is to add a torque contribution $h^{\prime} H a^{\prime} / 6 d_{\mathrm{c}}^{2}$ to $\Gamma_{\mathrm{e}}$ such that

$$
\Gamma_{\mathrm{e}}=\left|\boldsymbol{\Gamma}_{\mathrm{e}, \mathrm{core}}\right|+\sum_{i=1}^{N}{h^{\prime}}_{R, i} \frac{H a_{i}{ }^{\prime}}{6 d_{\mathrm{c}}{ }^{2}}
$$

where we consider $N$ asperities in contact with the membrane, of size $a_{i}^{\prime}$ and located at positions such that $h_{R, i}^{\prime}$ is the distance between point $E$ and the contact point of the asperity projected on the vertical plane $\left(O, \mathbf{e}_{R}, \mathbf{e}_{\mathrm{Z}}\right)$ as defined in Figure 1. Note that the contributions of other asperities not in contact with the membrane can be neglected due to the $d_{\mathrm{c}}^{-2}$ dependence of the torque they generate.

2.3. Hydrodynamics. Hydrodynamics come into play as a transport mechanism when particles are suspended in the fluid (step 1 ) and as a source of shear stress when particles touch the membrane (steps 2 and 3). A typical fluid velocity $V_{0}=1.2 \times 10^{-4} \mathrm{~m} \mathrm{~s}^{-1}$ can be obtained from the fluid flow rate through a single pore of the microsieve reported by Lin et al. Hydrodynamics and the particle trajectory are determined by the Reynolds and Stokes numbers. The particle Stokes number is the ratio of the particle viscous relaxation time, $a^{2} \rho_{\mathrm{p}} / 9 \mu_{\mathrm{f}}$, to a typical flow time scale $r_{\mathrm{p}} / V_{0}$ and is $O\left(10^{-5}\right)$. The particle Reynolds number $R e_{\mathrm{p}}=\rho_{\mathrm{f}} V_{0} a / \mu_{\mathrm{f}}$ is $O\left(10^{-4}\right)$ and the flow Reynolds number $R e_{\mathrm{f}}=\rho_{\mathrm{f}} V_{0} r_{\mathrm{p}} / \mu_{\mathrm{f}}$ is of the same order of magnitude. The fluid and particle motion are thus both noninertial, and the fluid flow obeys the Stokes equation.

2.3.1. Trajectory of a Suspended Particle. The first step of the present scenario of particle capture corresponds to the hydrodynamic transport of the particle until it first touches the membrane surface. In this study, a particle trajectory approach is used to describe the particle motion in the axisymmetrical flow cell depicted in Figure 1, which models the flow around a single pore in the membrane surface. This approach is aimed at obtaining probability distribution functions for particle deposition location as a function of the distance, $R$, to the pore center. Here, we will neglect the particle roughness effects and this assumption will be validated a posteriori by the results presented in subsection 3.1. 
For the low-Reynolds-number flow considered, a major difficulty lies in modeling the hydrodynamic interaction between the particle and the membrane surface. This was thoroughly studied for the geometry considered here by Wang et al. ${ }^{31}$ and Kao et al. ${ }^{32}$ This point was tackled by decomposing the problem into three distinct zeroReynolds-number flow problems: the pure translation (i) or rotation (ii) of a spherical particle in a quiescent fluid, and the imposed flow at infinity around a fixed particle (iii). Enhanced drag coefficients were introduced for each of these three cases, for any particle location, and summed to obtain the global drag force on the particle. These drag coefficients are "enhanced" in that they differ from the classical Stokes expression because they take the hydrodynamic interaction between the particle and the membrane into account. In the limit $R e_{\mathrm{f}} \ll 1$ and St $\ll 1$, the trajectory equation, modified to explicitly consider particle-membrane physicochemical interactions, reads:

$$
\frac{\mathrm{d} Z}{\mathrm{~d} R}=\frac{F_{R}^{\mathrm{t}}}{F_{Z}^{\mathrm{t}}} \frac{F_{Z}^{\mathrm{s}} V_{Z}+N_{\mathrm{adh}} F_{\mathrm{a}, Z}}{F_{R}^{\mathrm{s}} V_{R}+a \Omega F_{R}^{\mathrm{r}}+N_{\mathrm{adh}} F_{\mathrm{a}, R}}
$$

All the terms appearing in this equation are dimensionless. The $F_{j}^{i}$ terms are the enhanced drag coefficients and are functions of the particle location. The superscript refers to which of the three basic flows is considered ( $t$ for translation, $r$ for rotation, and $s$ for the flow past the fixed particle) and the subscript to the direction considered ( $Z$ or $R$, see Figure 1$)$. The term $\Omega$ denotes the sphere angular velocity and $\left(V_{R}, V_{Z}\right)$ the fluid velocity field. Additional information on these coefficients is given as Supporting Information. The $F_{\mathrm{a}, Z}$ and $F_{\mathrm{a}, R}$ terms are the $Z$ and $R$ components of the attractive vdW force computed numerically as described in subsection 2.2.1. The dimensionless ratio between the attractive $\mathrm{vdW}$ force and the particle-membrane hydrodynamic interaction appearing in the trajectory equation is called the adhesion number: $N_{\mathrm{adh}}=H / 6 \pi \mu_{\mathrm{f}} V_{0} a^{2}$, with its value being $2.6 \times 10^{-3}$ for the parameters of Lin et al. considered here. The particle trajectory eq 6 was integrated numerically using Runge-Kutta solvers implemented in Matlab.

2.3.2. Particle in Contact with the Membrane. Whenever a particle is in contact with the membrane or the pore edge, it is subject both to a hydrodynamic torque $\Gamma_{\mathrm{h}}$ due to the converging flow toward the pore opening and to an adhesion torque $\left(\Gamma_{\mathrm{a}}\right.$ or $\left.\Gamma_{\mathrm{e}}\right)$ resisting it. To obtain reliable values of the destabilizing hydrodynamic torque, the flow field around a fixed sphere of radius $a$ deposited close to the pore edge was first obtained by direct numerical simulations. Then, the overall torque $\Gamma_{\mathrm{h}}$ was obtained by numerical integration of the viscous stress tensor over the particle surface. Such numerical computations cannot be avoided in the situation of interest here because of the complex flow geometry and the lack of length scale separation between the particle size and the pore diameter: close to the pore edge, both shear rates $\mathrm{d} V_{R} / \mathrm{d} Z$ and $\mathrm{d} V_{Z} / \mathrm{d} R$ may provoke particle rolling and they vary over length scales comparable to or smaller than the particle size. Details on these numerical computations and the validation tests are given in the Supporting Information. The presence of the asperities on the surface of the particle was neglected in the aforementioned simulations. This is justified as follows: the hydrodynamic torque exerted on the spherical core (and around the contact point) is $\Gamma_{\mathrm{h}, \text { core }}$ $\propto \dot{\gamma} a^{3}$, while the contribution of all the $N$ asperities on the surface is $\Gamma_{\mathrm{h}, a s p} \propto N \dot{\gamma} a a^{\prime 2}$, where $\dot{\gamma}$ is some shear rate scale. $\Gamma_{\mathrm{h}, \text { asp }} / \Gamma_{\mathrm{h}, \text { core }}$ is therefore $O\left(N\left(a^{\prime} / a\right)^{2}\right) \equiv O(S)$, where the surface coverage of the asperities $S$ is $O\left(10^{-2}\right)$ in the present study as discussed in subsection 2.1.

\section{RESULTS AND DISCUSSION}

3.1. Particle Deposition. The particles were all launched at a distance $Z / R_{0}=2$ above the membrane surface, with varying radial positions $R_{i}$. Figure 2 a shows a typical example of a trajectory computed in the limiting case $N_{\text {adh }}=0$. As expected for a spherical particle subject to hydrodynamic interactions only, no deposition occurs due to a lubrication effect: the particle skims over the membrane surface before ultimately entering the pore. This is observed for all computed trajectories
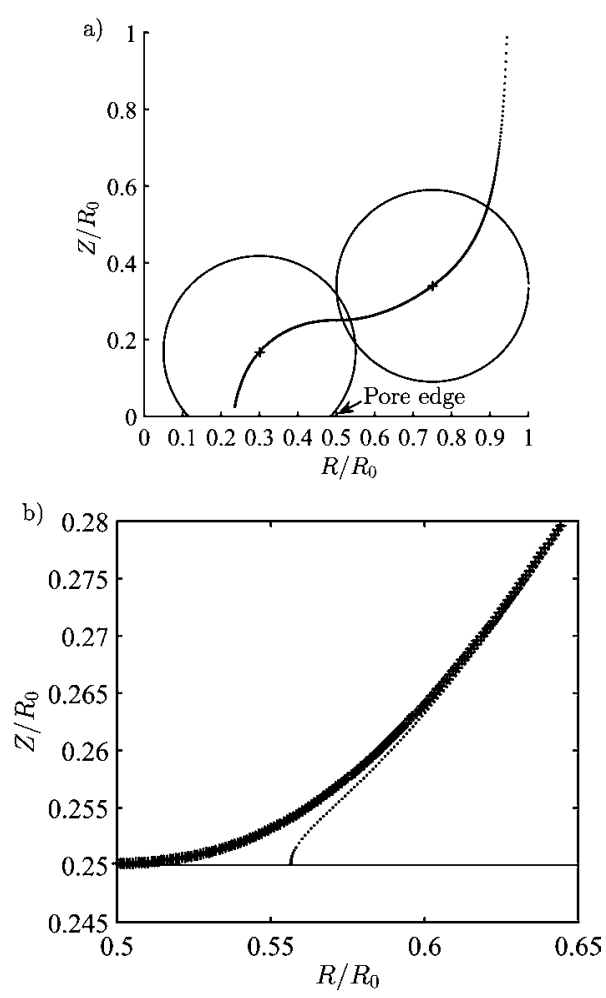

Figure 2. (a) Purely hydrodynamic trajectory $\left(N_{\text {adh }}=0, R_{i}=0.954 R_{0}\right)$ entering the pore. The particle is shown at two locations along the trajectory. The pore edge is located at $\left(R / R_{0}=0.5, Z / R_{0}=0\right)$; see also Figure 1. (b) Crosses, trajectory obtained with $N_{\text {adh }}=0$ and $R_{i}=$ $0.954 R_{0}$; dots, trajectory obtained with $N_{\text {adh }}=2.6 \times 10^{-3}$ and $R_{i}=$ $0.954 R_{0}$. The solid line at $Z / R_{0}=0.25$ shows the location reached by a particle center of mass when deposition occurs.

in that case. In Figure 2b, the same purely hydrodynamic trajectory is shown (crosses), along with a trajectory starting at the same $R_{i}$ but with $N_{\text {adh }}=2.6 \times 10^{-3}$ (dots). This trajectory deviates from the purely hydrodynamic one and finally "falls" onto the membrane as the normal component of the attractive $\mathrm{vdW}$ force diverges and overcomes the repulsive hydrodynamic interaction. This happens as soon as $Z / R_{0}<0.253$ here, that is, when the particle-membrane distance becomes smaller than about $30 \mathrm{~nm}$ (see Figure 2b). This behavior explains why neglecting the particle roughness is an acceptable assumption during the particle deposition stage. Surface roughness is known to make a contribution to particle-membrane hydrodynamic interaction only when a separation distance much smaller than the roughness length scale $a^{\prime}$ is reached, ${ }^{33}$ whereas, here, $a^{\prime}$ is of the order of $15 \mathrm{~nm}$. As far as the vdW interaction is concerned, the few bumps that are the closest to the membrane will determine the order of magnitude of the contribution of asperities to the vdW force. Denoting the spherical core-membrane distance by $d$, the latter contribution is $\mathrm{O}\left(\mathrm{Ha}^{\prime} /\left(d-\mathrm{a}^{\prime}\right)^{2}\right)$ while the contribution of the spherical core is $O\left(H a / d^{2}\right)$, so the contribution of asperities is non-negligible only when $d$ is smaller than $17 \mathrm{~nm}$ for the values of $a$ and $a^{\prime}$ considered here. To conclude, the contributions of the asperities to both the hydrodynamic and vdW forces become significant only when the trajectory of the smooth particle is already quite perpendicular to the membrane. Thus, the presence of asperities on the particle does not modify significantly the deposition locations found in the present trajectory analysis for a smooth sphere. 
We now turn to the analysis of particle trajectories leading to deposition, focusing on the location on the membrane where particle deposition occurs. From the knowledge of a discrete set of particle trajectories, a probability distribution function (pdf) for the radial deposition locations of particles, $f_{\mathrm{d}}(R)$, can be computed. Here, $f_{\mathrm{d}}(R)$ is normalized such that $f_{\mathrm{d}}(R) \mathrm{d} R$ is the fraction of the total number of deposited particles that deposit between $R$ and $R+\mathrm{d} R$. The pdf $f_{\mathrm{d}}(R)$ is displayed in Figure 3

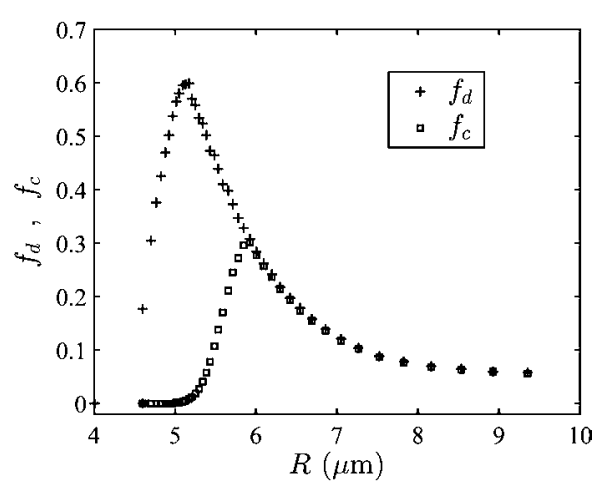

Figure 3. Probability distribution function for radial deposition locations of particles $f_{\mathrm{d}}(R)$ (crosses), normalized so that the area below $f_{\mathrm{d}}(R)$ is one, and for radial locations of particles that do not roll on the surface $f_{\mathrm{c}}(R)$ (squares).

(crosses) for $N_{\mathrm{adh}}=2.6 \times 10^{-3}$. The pdf shows a well-defined single maximum around $R \approx 5.1 \mu \mathrm{m}$ and does not exhibit the characteristic two-peaks shape of the experimental pdfs reported in Lin et al. This observation is robust as far as the value of the adhesion number is concerned (see the Supporting Information).

The present trajectory analysis predicts the locations at which particles first touch the membrane. This is not always their final capture position as they may still be able to start a rolling phase if the local shear flow is strong enough to overcome adhesion forces. This is the focus of the next section.

3.2. Particle Reentrainment. The second step in the present capture mechanism is the triggering of the rolling phase for some of the particles deposited as described in the previous section. For micrometer-sized particles, rolling is the main reentrainment mechanism, as will be discussed later. The occurrence of rolling is determined by the balance between a positive hydrodynamic torque $\Gamma_{h}$ acting on the particle, which tends to trigger rolling, and a negative stabilizing torque due to adhesion forces, with norm $\Gamma_{a}$. Rolling of a deposited particle will occur if $\Gamma_{\mathrm{h}}>\Gamma_{\mathrm{a}}$.

Dimensionless torque values $\Gamma_{\mathrm{h}} /\left(\mu_{\mathrm{f}} V_{0} R_{0}^{2}\right)$ obtained from the direct numerical simulations mentioned in subsection 2.3.2 are shown as a function of $s$ as squares in Figure 4. As expected, the torque value increases as $s$ decreases, i.e. when the particle is progressively positioned closer to the pore edge, with its center of mass being located above the membrane surface ( $s$ in the range $\left.\left[\begin{array}{ll}0 & 1\end{array}\right]\right)$. It continues to increase with $\theta$ when the particle center of mass is located above the pore opening while the particle-membrane distance remains fixed $(s$ in the range $[-1$ $0]$; see Figure 1), finally having a roughly 1 order of magnitude larger value than the value computed at $s=0.8$. Typical values for $\Gamma_{\mathrm{h}}$ are thus in the range $\approx O\left(10^{-17}-10^{-16}\right) \mathrm{N} \mathrm{m}$.

The probability of rolling at a radial position $R$ is $P\left(\Gamma_{a}<\right.$ $\left.\Gamma_{h}(R)\right)=P\left(a^{\prime}<a_{\mathrm{c}}^{\prime}(R)\right)$ where $a_{\mathrm{c}}^{\prime}(R)$ is the critical asperity size $a^{\prime}$ such that $\Gamma_{\mathrm{a}}=\Gamma_{\mathrm{h}}(R)$ with $\Gamma_{\mathrm{a}}$ given by relation 3 . The latter

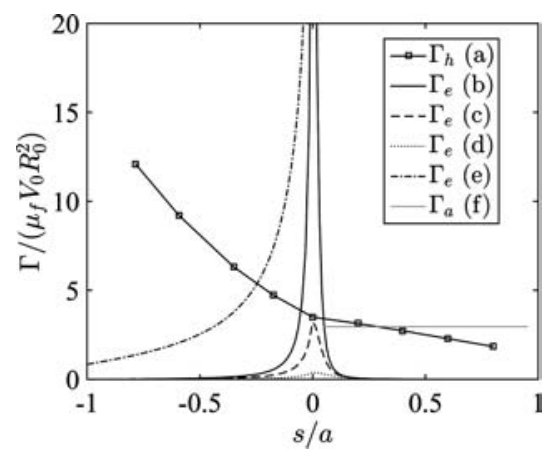

Figure 4. Hydrodynamic torque $\Gamma_{\mathrm{h}}$ and van der Waals torque $\Gamma_{\mathrm{e}}$ close to the pore edge as a function of $s$. (a) Squares, $\Gamma_{\mathrm{h}}$; (b) solid line, $\Gamma_{\mathrm{e}}$ for a smooth particle; (c) dashed line, $\Gamma_{\mathrm{e}}$ for a particle passing the pore edge while rolling on a $3 \mathrm{~nm}$ asperity; (d) dotted line, $\Gamma_{\mathrm{e}}$ for a particle passing the pore edge while rolling on a $15 \mathrm{~nm}$ asperity; (e) dashdotted line, $\Gamma_{\mathrm{e}}$ for the case shown on the leftmost sketch of Figure 5, with a $25 \mathrm{~nm}$ asperity (data restricted to $s<0$ only); (f) solid gray line, $\Gamma_{\mathrm{a}}$ computed using eq 3 , taking $a^{\prime}=15 \mathrm{~nm}$ (not normally distributed here) and $h=10 a^{\prime}$. All the torque values have been made dimensionless by dividing by $\mu_{\mathrm{f}} V_{0} R_{0}^{2}=1.2 \times 10^{-17} \mathrm{Nm}$, where $V_{0}=$ $1.2 \times 10^{-4} \mathrm{~ms}^{-1}$ is the fluid velocity used in the DNS and $R_{0}=10 \mu \mathrm{m}$ the flow cell radius. The contact distance $d_{\mathrm{c}}$ is $0.68 \mathrm{~nm}$ for curves (b)(e).

equality involves a nonlinear relation that has to be solved numerically for each position $R$. When $a^{\prime}$ is normally distributed, with mean value $\mu$ and standard deviation $\sigma$, the probability of rolling is the cumulative distribution function:

$$
\begin{aligned}
P_{\text {rolling }}(R) & =P\left(a^{\prime}<a_{\mathrm{c}}^{\prime}(R)\right) \\
& =\frac{1}{2}\left\{1+\operatorname{erf}\left[\frac{a_{\mathrm{c}}^{\prime}(R)-\mu}{\sqrt{ } 2 \sigma}\right]\right\}
\end{aligned}
$$

The pdf for location of particles that remain deposited on the membrane $f_{c}(R)$, that is, particles unable to start the rolling phase, is then:

$$
f_{\mathrm{c}}(R)=\left[1-P_{\text {rolling }}(R)\right] f_{\mathrm{d}}(R)
$$

It is represented as squares in Figure 3. The only parameter that was adjusted is $d_{\mathrm{c}}$. It was set to $0.68 \mathrm{~nm}$ so that $f_{\mathrm{c}}(R)$ exhibited a maximum located around $\mathrm{R}=6 \mu \mathrm{m}$, as reported for the outer capture position by Lin et al. Although slightly larger than usual values of the contact distance between solid surfaces, this value is physically sound and may be attributed to the hairy structure of latex particles at very small scale, the layer of polymer chains compressed between the asperity and the surface preventing closer contact. This value will be used throughout the article.

These results support the idea that some of the particles roll after deposition, because the hydrodynamic torque at their deposition location overcomes the adhesion torque. Particles that deposit close to the pore are more likely to start rolling, and consequently, the maximum of $f_{\mathrm{c}}(R)$ is shifted further away from the pore edge compared to the maximum of $f_{\mathrm{d}}(R)$. A similar shape for $f_{c}(R)$ is also obtained for values of $\mu$ and $d_{c}$ within $\pm 15 \%$ of the values considered here. The shift of the most probable capture position away from the pore entrance when the possibility of rolling after particle deposition is considered is thus a rather robust observation that is not expected to depend significantly on the details of the present 
modeling strategy and that is often overlooked in Lagrangian simulations of particle flows.

Such a competition between adhesion and hydrodynamic shear is also observed in "shear stress chambers" designed to study the resuspension of deposited particles as a function of a well controlled imposed hydrodynamic wall shear stress. ${ }^{34,35}$ Interestingly, using experimental data from Lorthois et al. ${ }^{35}$ for $2 \mu \mathrm{m}$ diameter bare sulfate latex particles initially deposited on a glass plate in such a device (very similar to the experimental case of Lin et al.), it is possible to estimate the adhesion torque that would be obtained for $5 \mu \mathrm{m}$ diameter particles. This requires the use of the scaling law of $\Gamma_{a}$ with the particle size $a$, $\Gamma_{a} \sim a^{2}$, obtained from the second term of the right-hand side of eq 3, which dominates the first one for the typical values of $a^{\prime}, h$, and $d_{c}$ considered here. The order of magnitude then obtained for the adhesion torque, $\approx O\left(10^{-17}\right) \mathrm{N} \mathrm{m}$, is similar to the one obtained numerically for $\Gamma_{\mathrm{h}}$. This strengthens the conclusion above about the likelihood of an heterogeneous triggering of particle rolling, obtained from the adhesion model used in the present study with realistic constitutive parameters.

Note that the present model only relies on a criterion to determine whether a deposited particle will be set in motion or not. A precise description of the subsequent particle motion along the membrane surface is neither within the scope of this paper nor necessary. However, for the following step of the scenario to be relevant, we need to check that a rolling particle does not lift off the membrane. The inertial lift force exerted on a moving rough particle in contact with a plane wall in a simple shear flow, made dimensionless by the particle weight corrected for buoyancy, scales as $R e^{2} / R e_{\mathrm{s}}$ where $R e=\dot{\gamma} a^{2} / \nu$ is a Reynolds number based on the shear rate $\dot{\gamma}$ and $R e_{s}=U_{s} a / \nu$ is a Reynolds number based on the particle settling velocity $U_{s}{ }^{36,37} \mathrm{Lift}$ effects on millimeter-size particles were observed experimentally for shear rate such that $R e^{2} / R e_{s}>1 .^{37}$ In the present case, lift would have to balance a particle-membrane adhesion force, which is much larger than the particle weight for micrometersized particles. Estimating the adhesion force due to the core of the particle only as $\mathrm{Ha} / \mathrm{a}^{\prime 2}$ provides a lower estimate for the adhesion force as it comes down to neglecting the additional contribution of roughness elements. Following the approach of Leighton and co-workers, ${ }^{36,37}$ the lift force, made dimensionless by the adhesion force, is found to scale as $\left(R e / N_{\mathrm{adh}}\right) \times\left(a^{\prime} / a\right)^{2}$, taking $\dot{\gamma} \sim V_{0} / a$. This ratio is typically $O\left(10^{-5}\right)$ in the present study: vdW interactions are sufficiently strong to prevent any lift effect for micrometer-sized particles, so that rolling particles do not lift off the membrane.

3.3. Particle Capture. The third step in the present capture mechanism concerns only particles that are reentrained by the flow after deposition, by rolling on the membrane surface. Again, the specific geometry of the problem comes into play. The main idea is that a particle rolling on the surface and approaching the pore edge will experience a localized stabilizing torque of norm $\Gamma_{e}$ when it reaches the edge. This is due to the strong symmetry breaking in the $\mathrm{vdW}$ force distribution close to the contact region. An inertialess particle will therefore be captured on the pore edge, at the coordinate $s$ for which $\Gamma_{\mathrm{e}}(s)=$ $\Gamma_{\mathrm{h}}(s)$.

As a reference problem and to illustrate the physics involved, we first consider the case of a nondeformed, smooth, spherical particle rolling on a smooth surface. Values for $\Gamma_{\mathrm{e}} /\left(\mu_{\mathrm{f}} V_{0} R_{0}^{2}\right)$ computed as described in subsection 2.2.3 are shown as a function of $s$ by the solid line in Figure 4. When $s>0.1, \Gamma_{\mathrm{e}}$ is negligible but a sudden increase is observed when the particle comes close to the pore edge $(s \rightarrow 0)$. There is an increase in torque values of 2 orders of magnitude between $s=0.1$ (i.e., $\mathrm{R}$ $=5.1 \mu \mathrm{m})$ and $s=0(\mathrm{R}=5.0 \mu \mathrm{m})$ as the particle starts to "feel" the symmetry breaking induced by the pore edge on the vdW interactions. When $s$ becomes negative, the decay in the torque value is also very sharp: for $s=-0.6$ (corresponding to $\theta=$ $125^{\circ}$; see Figure 1), the torque value is 2 orders of magnitude smaller than the typical destabilizing torque values $\Gamma_{\mathrm{h}}\left(\Gamma_{\mathrm{e}} \approx\right.$ $\left.0.05 \times \mu_{\mathrm{f}} V_{0} R_{0}^{2}\right)$. The maximal torque value obtained for $s=0$ depends strongly on the particle-membrane distance, $d$, considered. By varying $d$, it is found that, as soon as $d$ is smaller than a few nanometers, this maximum becomes larger than the typical values computed for the hydrodynamic torque $\left(\Gamma_{\mathrm{h}} /\left(\mu_{\mathrm{f}} V_{0} R_{0}^{2}\right) \approx 3\right)$. This demonstrates that the phenomenon of particle capture on a pore edge swept by a flow is quite robust for typical microfluidics flow velocities.

Although the physical idea exposed above remains relevant when a spherical particle covered with hemispherical asperities is considered, the exact description is more complex as it depends on the relative locations of the pore edge and the closest roughness elements on the particle surface. For the sake of conciseness and clarity, only two situations will be discussed. The first is the case where the particle passes the pore edge while rolling on an asperity (see the rightmost sketch on Figure 5 ). Then, both the particle and the asperity contribute to the

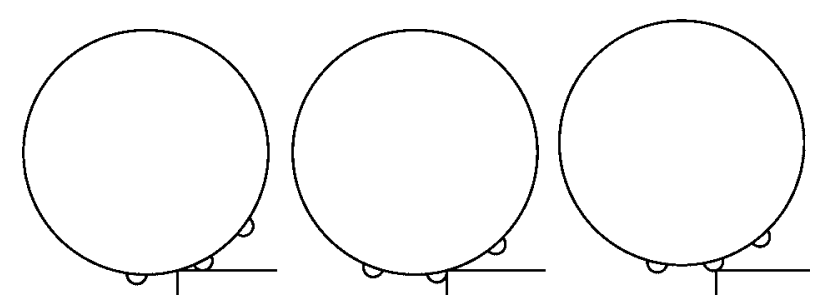

Figure 5. Three examples of asperity configurations. Left: asperity stabilizes the particle on the membrane. Middle: asperity destabilizes the particle. Right: asperity shifts the particle from the membrane.

torque, the maximum of which remains localized around $s=0$ as both the particle and asperity "feel" the dissymmetry due to the pore edge. For an asperity radius smaller than about $30 \mathrm{~nm}$, the main contribution to the torque is due to the particle, the small effect of an asperity being mainly to shift the particle away from the surface. For an asperity radius smaller than $3 \mathrm{~nm}$ (dashed line on Figure 4), the localized torque obtained remains stronger than the hydrodynamic one, which causes the inertialess particle to be captured around $s=0$. For larger asperities (e.g., dotted line on Figure 4 for a $15 \mathrm{~nm}$ asperity), $\Gamma_{\mathrm{e}}$ is not strong enough to stop the particle from rolling into the pore. Second, when no asperity is in contact with the pore edge while the particle rolls over it, any detailed computation of $\Gamma_{\mathrm{e}}$ requires a precisely prescribed geometry (relative position of pore edge and of the closest asperities, with two examples being provided on the two leftmost sketches of Figure 5). If some asperities touch the membrane upstream of the pore edge, particle capture at $s<0$ locations is promoted by the last term in relation 5. Considering some asperity positioned as depicted on the leftmost sketch of Figure 5, the distance $h_{R}^{\prime}$ involved in eq 5 is uniquely (although not explicitly) defined as a function of $s$ from simple geometrical considerations. It is rather expected that at least two such asperities will be in contact with the membrane since the presence of only one would lead to particle rolling in the azimuthal direction until a second contact 
point is reached. Besides, it is not very likely that a third asperity is also exactly in, or close to, contact with the membrane given that their spacing is $O\left(10 a^{\prime}\right)$. The contribution of such a third asperity is thus neglected, leading to an approximate expression for the total stabilizing vdW torque:

$$
\Gamma_{\mathrm{e}}(s) \simeq\left|\Gamma_{\mathrm{e}, \text { core }}(s)\right|+2 h_{R}^{\prime}(s) \frac{H a^{\prime}}{6 d_{\mathrm{c}}^{2}}
$$

The prediction 9 is depicted as a dash-dotted line for a large asperity with $a^{\prime}=25 \mathrm{~nm}$ in Figure 4. The torque values obtained are larger than hydrodynamic ones for pore/particle/ asperity configurations corresponding to angles $\theta$ up to $104^{\circ}$, that is, for a particle center of mass located down to $R \simeq 4 \mu \mathrm{m}$, well above the pore opening. Detailing the exact rolling step to such a final capture location is beyond the scope of this paper but the previously computed example clearly shows that particles may be finally captured with their center of mass located well above the pore opening, especially if the particles present some degree of surface roughness. Note, however, that the strong $\mathrm{vdW}$ torque $\Gamma_{\mathrm{e}, \text { core }}$ of a smooth particle is in itself sufficient to effectively arrest the particles on the pore edge for the present hydrodynamic and physicochemical conditions.

Consequently, after this last step, the pdf for the radial location of captured particles will exhibit two preferential locations: an "outer" one due to particles that did not roll after deposition (around $R=6 \mu \mathrm{m}$ for the case shown in Figure 3) and an "inner" one due to particles that rolled on the surface after deposition and were finally captured close to the pore edge by the stabilizing vdW torque $\Gamma_{\mathrm{e}}$. It is important to note that this population of captured particles will be supplemented by the particles that deposited directly very close to the pore edge and did not roll because of the existence of $\Gamma_{\mathrm{e}}$. Therefore, the proposed three-step scenario provides an explanation for Lin et al.'s experimental observations where two such preferential locations were reported (for $5.2 \mu \mathrm{m}$ and also 2.1 $\mu \mathrm{m}$ diameter latex particles). Even if approximations have been made to render the model tractable (expressions 3 and 9, Hamaker additivity,...), the comments and arguments exposed above suggest that improvements of the modeling would not contradict this conclusion. Indeed, refining the contribution of asperities in relation 3 might shift the position of the outer capture position inward or outward, and the addition of contributions of other asperities in relation 9 would only alter the efficiency of capture at the inner position. Therefore, although quantitative predictions on the exact capture positions and the capture rate at these positions are probably out of reach of the present modeling, the qualitative picture of the existence of two preferential capture positions, one being counterintuitively above the pore opening, is robust in the present three-step scenario.

\section{CONCLUSION}

Recent developments in the physics of particle capture, often in the light of recent carefully designed microfluidics modeling experiments, reveal the appearance of bridges or arches of particles across the entrances of pores. The creation of these bridges may be explained if at least some particles are arrested on the pore edges to initiate the arches, as observed in some experiments. In this paper, we have shown that such an observation can be predicted with a model including particle deposition on the membrane, a subsequent rolling phase for some of the deposited particles, and a final arrest on the pore edge due to the onset of a stabilizing adhesion torque when the particle approaches this edge. This is a clear improvement over previous approaches that neglected particle reentrainment and overlooked the existence of a large stabilizing vdW adhesion torque close to the pore edge, thus failing to describe the counterintuitive capture location over the pore edge.

In view of the order of magnitude of the adhesion and hydrodynamic torques estimated earlier and their scaling with particle size $\left(\Gamma_{\mathrm{a}}\right.$ and $\Gamma_{\mathrm{e}} \sim a^{2}$, and $\left.\Gamma_{\mathrm{h}} \sim a^{3}\right)$, we expect this scenario to be quite generic for particles in the micrometer size range $(a \approx 1-10 \mu \mathrm{m})$, as often encountered in microfluidic studies of particle capture, when both particle-Reynolds and particle-Stokes numbers are small. Larger particles may not be captured at all and smaller ones may not roll after deposition or may leave the surface due to thermal effects.

The present study also highlights the importance of modeling particle adhesion on the surface, as tiny, nanometer scale, details can have a dramatic effect on micrometer-sized particles capture. Despite this difficulty, this work suggests that such a touching-rolling-halting mechanism should be implemented in Lagrangian numerical codes intended to predict pore clogging phenomena. In addition, predictions of capture rates and capture locations will clearly depend on the statistics of surface properties, which suggests that statistical approaches dealing with this point cannot be avoided, as reported by Henry and co-workers. ${ }^{30,38}$

\section{AUTHOR INFORMATION}

\section{Corresponding Author}

*E-mail: paul.duru@imft.fr.

\section{Notes}

The authors declare no competing financial interest.

\section{ACKNOWLEDGMENTS}

This work was performed using HPC resources from CALMIP (Project p1114) and GENCI (IDRIS/CINES/TGCC, Grant x2015097003).

\section{REFERENCES}

(1) Seinfeld, J. H.; Pandis, S. N. Atmospheric chemistry and physics: from air pollution to climate change; John Wiley \& Sons: New York, 2012.

(2) Espinosa-Gayosso, A.; Ghisalberti, M.; Ivey, G. N.; Jones, N. L. Particle capture and low-Reynolds-number flow around a circular cylinder. J. Fluid Mech. 2012, 710, 362-378.

(3) Rushton, A.; Ward, A. S.; Holdich, R. G. Solid-liquid filtration and separation technology, 2nd ed.; John Wiley \& Sons: New York, 2000.

(4) Bacchin, P.; Marty, A.; Duru, P.; Meireles, M.; Aimar, P. Colloidal surface interactions and membrane fouling: investigations at pore scale. Adv. Colloid Interface Sci. 2011, 164 (1), 2-11.

(5) Agbangla, G. C.; Climent, E.; Bacchin, P. Experimental investigation of pore clogging by microparticles: Evidence for a critical 
flux density of particle yielding arches and deposits. Sep. Purif. Technol. 2012, 101, 42-48.

(6) Agbangla, G. C.; Bacchin, P.; Climent, E. Collective dynamics of flowing colloids during pore clogging. Soft Matter 2014, 10 (33), 6303-6315.

(7) Ramachandran, V.; Fogler, H. S. Plugging by hydrodynamic bridging during flow of stable colloidal particles within cylindrical pores. J. Fluid Mech. 1999, 385, 129-156.

(8) Sharp, K. V.; Adrian, R. J. On flow-blocking particle structures in microtubes. Microfluid. Nanofluid. 2005, 1 (4), 376-380.

(9) Mustin, B.; Stoeber, B. Deposition of particles from polydisperse suspensions in microfluidic systems. Microfluid. Nanofluid. 2010, 9 (45), 905-913.

(10) Lin, J.; Bourrier, D.; Dilhan, M.; Duru, P. Particle deposition onto a microsieve. Phys. Fluids 2009, 21 (7), 073301.

(11) Wyss, H. M.; Blair, D. L.; Morris, J. F.; Stone, H. A.; Weitz, D. A. Mechanism for clogging of microchannels. Phys. Rev. E 2006, 74, 061402.

(12) Sauret, A.; Barney, E. C.; Perro, A.; Villermaux, E.; Stone, H. A.; Dressaire, E. Clogging by sieving in microchannels: Application to the detection of contaminants in colloidal suspensions. Appl. Phys. Lett. 2014, 105 (7), 074101.

(13) Wu, X.; van de Ven, T. G. M. Characterization of hairy latex particles with colloidal particle scattering. Langmuir 1996, 12 (16), 3859-3865.

(14) Seebergh, J. E.; Berg, J. C. Evidence of a hairy layer at the surface of polystyrene latex particles. Colloids Surf., A 1995, 100, 139-153.

(15) Considine, R. F.; Hayes, R. A.; Horn, R. G. Forces measured between latex spheres in aqueous electrolyte: Non-DLVO behavior and sensitivity to dissolved gas. Langmuir 1999, 15 (5), 1657-1659.

(16) Suresh, L.; Walz, J. Y. 1996. Effect of surface roughness on the interaction energy between a colloidal sphere and a flat plate. J. Colloid Interface Sci. 1996, 183 (1), 199-213.

(17) Israelachvili, J. Intermolecular and surface forces, 3rd ed.; Academic Press: London, 2011.

(18) French, R. H.; Parsegian, V. A.; Podgornik, R.; Rajter, R. F.; Jagota, A.; Luo, J.; Zemb, T. Long range interactions in nanoscale science. Rev. Mod. Phys. 2010, 82 (2), 1887-1944.

(19) Argento, C.; Jagota, A.; Carter, W. C. Surface formulation for molecular interactions of macroscopic bodies. J. Mech. Phys. Solids 1997, 45 (7), 1161-1183.

(20) Yang, P.; Qian, X. 2009. A general, accurate procedure for calculating molecular interaction force. J. Colloid Interface Sci. 2009, 337 (2), 594-605.

(21) Bhattacharjee, S.; Chen, J. Y.; Elimelech, M. DLVO interaction energy between spheroidal particles and a flat surface. Colloids Surf., A 2000, 165, 143-156.

(22) Martines, E.; Csaderova, L.; Morgan, H.; Curtis, A. S. G.; Riehle, M. O. DLVO interaction energy between a sphere and a nanopatterned plate. Colloids Surf., A 2008, 318 (1-3), 45-52.

(23) Bowen, W. R.; Filippov, A. N.; Sharif, A. O.; Starov, V. M. A model of the interaction between a charged particle and a pore in a charged membrane surface. Adv. Colloid Interface Sci. 1999, 81, 35-72.

(24) Bhattacharjee, S.; Sharma, A. Lifshitz-van der Waals energy of spherical particles in cylindrical pores. J. Colloid Interface Sci. 1995, 171 (2), 288-296.

(25) Hallez, A. Analytical and numerical computations of the van der Waals force in complex geometries: Application to the filtration of colloidal particles. Colloids Surf., A 2012, 414, 466-476.

(26) Rumpf, H. Particle technology; Chapman \& Hall: London, 1990.

(27) Rabinovich, Y. I.; Adler, J. J.; Ata, A.; Singh, R. K.; Moudgil, B. $\mathrm{M}$. Adhesion between nanoscale rough surfaces: I. Role of asperity geometry. J. Colloid Interface Sci. 2000, 232 (1), 10-16.

(28) Henry, C.; Minier, J. P.; Lefevre, G. Towards a description of particulate fouling: from single particle deposition to clogging. Adv. Colloid Interface Sci. 2012, 185, 34-76.

(29) Prokopovich, P.; Starov, V. Adhesion models: From single to multiple asperity contacts. Adv. Colloid Interface Sci. 2011, 168 (1), $210-222$.
(30) Henry, C.; Minier, J. P. Progress in particle resuspension from rough surfaces by turbulent flows. Prog. Energy Combust. Sci. 2014, 45, $1-53$.

(31) Wang, Y.; Kao, J. N.; Weinbaum, S.; Pfeffer, R. On the inertial impaction of small particles at the entrance of a pore including hydrodynamic and molecular wall interaction effects. Chem. Eng. Sci. 1986, 41 (11), 2845-2864.

(32) Kao, J. N.; Wang, Y.; Pfeffer, R.; Weinbaum, S. A theoretical model for nucleopore filters including hydrodynamic and molecular wall interaction effects. J. Colloid Interface Sci. 1998, 121 (2), 543-557.

(33) Smart, J. R.; Leighton, D. T., Jr. Measurement of the hydrodynamic surface roughness of noncolloidal spheres. Phys. Fluids A 1989, 1 (1), 52-60.

(34) Lorthois, S.; Schmitz, P.; Anglés-Cano, E. Experimental study of fibrin/fibrin-specific molecular interactions using a sphere/plane adhesion model. J. Colloid Interface Sci. 2001, 241 (1), 52-62.

(35) Lorthois, S.; Schmitz, P.; Houi, D.; Anglés-Cano, E. Experimental study of fibrin embolization under shear flow. J. Adhes. 2000, 72 (2), 229-239.

(36) Krishnan, G. P.; Leighton, D. T., Jr. Inertial lift on a moving sphere in contact with a plane wall in a shear flow. Phys. Fluids 1995, 7, $2538-2545$.

(37) King, R. K.; Leighton, D. T., Jr. Measurement of the inertial lift on a moving sphere in contact with a plane wall in a shear flow. Phys. Fluids 1997, 9, 1248-1255.

(38) Henry, C.; Minier, J. P.; Lef èvre, G. Numerical study on the adhesion and reentrainment of nondeformable particles on surfaces: the role of surface roughness and electrostatic forces. Langmuir 2012, 28 (1), 438-452. 